\title{
Access to Health Information
}

\section{Findings from the Section on Access to Health Information}

\author{
A. Gaudinat, Managing Editor for the IMIA Yearbook Section on Access to Health Information \\ Health on the Net Foundation, SIM/HUG, Geneva, Switzerland
}

\section{Introduction}

In 2006, according to a Pew [1] study, $80 \%$ of the American Internet users have searched health information on the Web. Most of them used a general search engine to reach this information and only $15 \%$ of these seekers indicated checking source and date of the online health information found. From a professionals perspective, Internet and web based medical information is also widely popular amongst physicians [2] and can allow them to make a better final decision [3]. Main human challenges in access to health information are well known, giving an equivalent, efficient and trustworthy access for all independent of the geographical location, economical situation, cultural aspect and educational level [4] (so called universal access[5]). From a continuous advance in Internet and search technology during the previous decade, access to health information has never as good, for both individual and professional. Furthermore, despite this technological improvement, challenges in technology still remain, in order to allow users to find their way amongst this huge volume of available information, to recognize the quality of this information [6] and to improve access for all types of population. All these challenges are not related to health only, but are more critical in this domain. As already noted in the Yearbook 2007, a promise and observed trend is the patient empowerment, thanks to current technologies. In particular, the Web 2.0 [7] should allow giving to the patient more implication and access to health information and especially to their personal file. Effort in consumer health domain, particularly for the lay user, such as the excellent Consumer Health Vocabulary Initiative [8], illustrate this trend to improve access for all types of population.

\section{Best Paper Selection}

The best paper selection of articles for the section 'Access to health information' in the IMIA Yearbook 2008 follows the tradition of previous Yearbooks. As a result of a comprehensive reviewing process, four excellent and original papers were selected in this section, a brief content summary of which can be found in the appendix of this paper.

These four papers give an overview of important trends and challenges in this field but don't cover all the aspects of the broadness of this field. Access to health information is primary (but not only) related to information search technology applied to health and this selection presents different aspects of the information search which are the user decision making, unconscious behavior, way to display results and multi- 
lingualism access. Two of the papers are original studies about user behavior during access to health information. One is patient-oriented since it describes how patients build their decision and make their final opinion during a search about a specific medical subject [9]. The second study deals with the possible biases made by information seekers [10] (professionals and non-clinician students). The two other papers describe interesting health search engine applications where the first presents different ways to access MEDLINE by using clusters with MeSH keywords [11] and the second deals with cross-lingual information retrieval based on morphological resources [12]. These two last papers have an evaluation section which allows better appreciation of the quality of their approaches.

\section{Conclusions and Outlook}

Four excellent articles are the results of the selection process for this yearbook section. This selection indicates that users are the main subject of interest when studying the access to health information. However the topic of this section is very broad and other trends presented during other years were not well represented this year or belong also to other sections such as the education and consumer section. For instance, Web 2.0 will be a heavy trend during the coming years concerning the access to health information. This 'soft revolution' will empower patients to be more active and will definitely change the access paradigm. So researchers will have to design new studies to observe how users access and assess the health information in the social Web, and to produce efficient interface to retrieve this information in a safe and trustworthy environment.

Table 1 Best paper selection of articles for the IMIA Yearbook of Medical Informatics 2008 in the section 'Access to Health Information'. The articles are listed in alphabetical order of the first author's surname.

Section

Access to Health Information

- Daumke P, Kornel M, Poprat M, Schulz S. Biomedical Information Retrieval Across Languages. Informatics for Health and Social Care 2007;32:131-47

- Lau A, Coiera E. Do People Experience Cognitive Biases while Searching for Information? JAMIA 2007;14:599-608.

- Lin Y, Li W, Chen K, Liu Y. A Document Clustering and Ranking System for Exploring MEDLINE. JAMIA,2007;14:651-61.

- Sillence E, Briggs P, Harris PR, Fishwick L. How do patients evaluate and make use of online health information? Sci Med 2007;May;64(9):1853-62.

\section{Acknowledgement}

I greatly acknowledge the support of Martina Hutter and Mayoni Ranasinghe as well as of the reviewers in the selection process of the IMIA Yearbook.

\section{References}

1. Fox S. Online Health Search 2006, Pew Internet \& American Life Project;2006.

2. Westbrook J, Coiera E, Gosling S. Do Online Information Retrieval Systems Help Experienced Clinicians Answer Clinical Questions? J Am Med Inform Assoc 2005;12:315-21.

3 Hesse BW, Nelson DE, Kreps GL, Croyle RT, Arora NK, Rimer BK, et al. Trust and Sources of Health Information: The Impact of the Internet and Its Implications for Health Care Providers: Findings From the First Health Information National Trends Survey. Arch Intern Med 2005;165:2618-24.

4. McCray A. Promoting Health Literacy. J Am Med Inform Assoc 2005;12:152-63.

5. Godlee F, Pakenham-Walsh N, Ncayiyana D, Cohen B, Packer A. Can we achieve health information for all by 2015? The Lancet 2004;364(9430):295-300.

6. Boyer C, Grabar N, Gaudinat A, Geissbuhler A. Models of trust for health websites. Medinfo 2007, Workshop, Brisbane, Australia; 2007.

7. Boulos M, Maramba I, Wheeler S. Wikis, blogs and podcasts: a new generation of web-based tools for virtual collaborative clinical practice and education. BMC Med Educ 2006;6:41.

8. Zeng Q, Tse T, Divita G, Keselman A, Crowell, Browne A, et al. Term Identification Methods for Consumer Health Vocabulary Development. J Med Internet Res 2007;9(1)

9. Sillence E, Briggs P, Harris PR, Fishwick L. How do patients evaluate and make use of online health information? Sci Med 2007;May;64(9):1853-62.

10. Lau A, Coiera E. Do People Experience Cognitive Biases while Searching for Information? JAMIA 2007;14:599-608.

11. Lin Y, Li W, Chen K, Liu Y. A Document Clustering and Ranking System for Exploring MEDLINE. JAMIA 2007;14:651-61.

12. Daumke P, Kornel M, Poprat M, Schulz S. Biomedical Information Retrieval Across Languages. Informatics for Health and Social Care 2007;32:131-47
Correspondence to:

Arnoud Gaudinat

Health on the Net Foundation

SIM/HUG

24 rue Micheli-du-Crest

Geneva, Switzerland

Tel: +41223724843

Fax: +41 223728885

E-mail: arnaud.gaudinat@healthonnet.org

Appendix: Content Summaries of Selected Best Papers for the IMIA Yearbook 2008, Section Access to Health Information*

\section{Sillence E, Briggs P, Harris PR, Fishwick L. How do Patients Evaluate and Make Use of Online Health Information? \\ Sci Med 2007;May;64(9): 1853-62}

The behavior of patients towards online health information is a crucial point of interest, and in particular what will make them decide whether or not to trust the content they find online. This study tries to identify what the main factors are, which conduct patients to

* The complete papers can be accessed in the Yearbook's full electronic version, provided that permission has been granted by the copyright holder(s) 
make a final decision when consulting online health information. Fifteen women were observed as they made decisions concerning the menopause and hormone replacement therapy (HRT), during a 3-stage study (log analysis, 6 month diaries and a final interview). Authors found that website design plays the main role to keep or reject health information and is independent of the content quality. Furthermore pharmaceutical industry websites or sponsored websites (recognized as high-quality content) can be directly rejected due to their supposed partiality. Finally women with a 'scientist-like' profile prefer to discuss with friends, family and especially physicians before making a final decision.

\section{Lau A, Coiera E}

Do People Experience Cognitive Biases while Searching for Information? JAMIA,2007;14:599-608

This original article proposes to measure the importance of cognitive biases when people search online information. This study is composed of two parts, a retrospective analysis (75 clinicians, answering questions on clinical scenarios) and a prospective experiment (227 undergraduate students who used the same search engine). Four possible cognitive biases are studied: the anchoring effect, the order effect, the exposure effect, and the reinforcement effect. The results show evidence that people can experience cognitive biases while searching for information. In decreasing order, the main cognitive biases which influence the search are anchoring effect, order effect and exposure effect. These findings can be useful to better design search engines and to improve the quality of decision making during and after the search.

\section{Lin Y, Li W, Chen K, Liu Y}

\section{A Document Clustering and Ranking System for Exploring MEDLINE}

JAMIA 2007;14:651-61

Access to information in the health domain also depends on the way in which search results are presented to the user, especially when one query can give several thousands of interesting results. This article presents a text mining system based on Pubmed queries, which provides an interesting citations clustering and ranking system, as well as a set of keywords and MeSH terms to describe the common theme of each cluster. Authors have evaluated several ranking functions which were citation count per year (CCPY), citation count (CC), and journal impact factor (JIF). The results show that CCPY outperforms the two other ranking functions. Authors conclude that this system succeeds in building informative clusters for MEDLINE database which are revealed by the keywords and $\mathrm{MeSH}$ terms extracted from the documents in each cluster.
Daumke P, Kornel M, Poprat M, Schulz S

Biomedical Information Retrieval Across Languages

Informatics for Health and Social Care 2007;32:131-47

Cross-language information retrieval (CLIR) is an important research topic and challenge because of the unevenness of contents available in various languages, the prominence of the English language on the Web and the difficulties encountered for automatic translation. Authors present a new dictionary-based approach to biomedical CLIR. The presented system is strongly guided by morphology and semantic resources in six European languages to improve its precision. Mediation between query and document is based on morphological fragmenting of words and list of word-n-grams, generated from large monolingual corpora. The translations are sent to a standard Internet search engine (Google). An evaluation has been conducted on the OHSUMED corpus to measure the quality of the CLIR where the best retrieval performance outcomes of $88 \%$ of the monolingual baseline performance for German. Results for Spanish, French and Swedish are lower, certainly due to the lack of data and resources for these languages, while Portuguese yields promising results. 